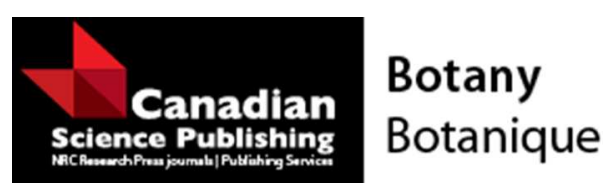

\title{
Using woody genera for phytogeographic regionalization at a medium scale: A case study of Italy
}

\begin{tabular}{|r|l|}
\hline Journal: & Botany \\
\hline Manuscript ID & cjb-2015-0234.R2 \\
\hline Manuscript Type: & Article \\
\hline Complete List of Authors: & $\begin{array}{l}\text { Abbate, Giovanna; University of Rome La Sapienza, Biologia Ambientale } \\
\text { Scassellati, Elisabetta; Università di Roma La Sapienza } \\
\text { Bonacquisti, Sandro; Università di Roma La Sapienza, Department } \\
\text { Environmental Biology } \\
\text { Iberite, Mauro; Università di Roma La Sapienza } \\
\text { Latini, Marta; Università di Roma La Sapienza } \\
\text { Giuliani, Alessandro; Istituto Superiore di Sanità }\end{array}$ \\
\hline Keyword: & $\begin{array}{l}\text { floristic pattern, geographic gradient, higher-taxon approach, native woody } \\
\text { flora, orographic index }\end{array}$ \\
\hline & \\
\hline
\end{tabular}


Using woody genera for phytogeographic regionalization at a medium scale: A case study of Italy

Giovanna Abbate $^{\mathrm{a} *}$, Elisabetta Scassellati ${ }^{\mathrm{a}}$, Sandro Bonacquisti ${ }^{\mathrm{a}}$, Mauro Iberite ${ }^{\mathrm{a}}$, Marta Latini ${ }^{\mathrm{a}}$, Alessandro Giuliani $^{\mathrm{b}}$

${ }^{\mathrm{a} D}$ Dipartimento di Biologia Ambientale, Sapienza Università di Roma, P.le Aldo Moro 5, 00185 Roma, Italy ${ }^{b}$ Dipartimento Ambiente e Salute, Istituto Superiore di Sanità, Roma, Italy

*Corresponding author: Email: giovanna.abbate@uniroma1.it, Phone/Fax +39 0649912828 


\begin{abstract}
We present a phytogeographic regionalization based on native woody flora, identifying the most useful taxonomic level, geographic variables, and orographic pattern, selecting Italy as a case study. We generated seven distance matrices among the 20 administrative regions, and using Pearson's correlation coefficients and PCA, we verified whether distances between regions were invariant across the different sampling strategies. Once this invariance was established, we focused on genera representation. We defined two orographic indices and performed Kruskal-Wish multidimensional scaling and K-means clustering to assess Italy's phytogeographic regionalization. A major North-South and a minor East-West gradient described the relationships between regions. Floristic diversity was strongly correlated with the region's orography, with hills being the most important orographic feature that increases plant diversity; the effect of the orographic patterns was independent from the geographic clines observed. Despite the coarse scale, our phytogeographic regionalization comprising six clusters (variables $=133$ woody genera) was consistent with previous ones based on the endemic flora (variables $=1371$ units) or on bioclimatic approaches. In particular, the phytogeographic uniqueness of Northern and peninsular Italy, and of Sardinia Island, was confirmed. Next step will be to test our method at a finer scale.
\end{abstract}

Keywords: floristic pattern, geographic gradient, higher-taxon approach, native woody flora, orographic index. 


\section{Introduction}

The distribution of species through time and space remains a central research theme at all scales (Heikinheimo et al. 2007, Rueda et al. 2010). Biogeographic regions, which are important generalisations of the organisation of life on Earth based on past and current physical and biological components, provide the spatial framework for studying many basic questions in historical and ecological biogeography, systematics, and evolutionary biology (Kreft and Jetz 2010). Ecological biogeography, in particular, seeks the causes explaining distribution patterns in short temporal scales (Posadas et al. 2006). Biogeographic regions have been used in macroecological studies of species richness as well as for applied ecology purposes (Olson et al. 2001). In addition, the delineation of biogeographic regions represents a useful tool in conservation planning and management, as recognised by classic and applied biogeographers (Heikinheimo et al. 2007, Rueda et al. 2010, Whittaker et al. 2013).

Early biogeographic regionalizations at the continental scale were based on vegetation zones or relationships between climate, plants, and animals (von Humboldt 1806, de Candolle 1855, Wallace 1876). Later, Takhtajan (1986) proposed a system of phytochoria (phytogeographic units) based on plant endemism levels. The currently accepted European biogeographic map was developed by Rivas-Martinez et al. (2004). As for Italy, the regionalization proposed by Giacomini (1958) is still widely accepted as the most consistent one. Peruzzi et al. (2014) recently described floristic affinities between the 20 administrative regions in Italy, by using a dataset comprising 1371 endemic species and subspecies.

Recent broad-scale biogeographic studies employed quantitative methods, as illustrated by Kreft and Jetz (2010). They were mainly based on climatic and geographic parameters, and many groups of organisms were used as biodiversity predictors and processed using grid maps (Field et al. 2007, Veetas and Ferrer-Castán 2008, Rueda et al. 2010). Valuable insights into biogeographic patterns have been recently provided using natural habitats (Divíšek et al. 2014).

The biogeographic regions map published by the European Commission in the context of Natura 2000 Network (European Environmental Agency, 2012) reported three coarsely delimited regions in Italy: Alpine, Continental, and Mediterranean. In addition to climatic, geological, and geomorphological data, Italy's ecoregional classification (Blasi et al. 2014) uses different biogeographic categories to distinguish between the different ecological units. 
Shortcuts for quantifying species patterns at different scales have recently assumed a great interest not only in biogeography but also in conservation planning and management. Woody flora, trees in particular, have been shown to be important biodiversity surrogates for highly heterogeneous areas such as the Iberian Peninsula (Veetas and Ferrer-Castán 2008); as concerns Italy, woody plant richness has been shown, at a medium scale, as a good predictor of the overall floristic richness (Abbate et al. 2015). Furthermore, evidence suggests woody flora and trees as the biotic drivers of animal distribution by providing a habitat structure, as measured by tree distribution, appearing to have a strong influence on the biogeographic structure of many animal groups (Rueda et al. 2010). Based on the assumption that family and genus identification is easier than species identification, some authors have used a higher-taxon approach as a valid alternative to species in several groups of organisms (La Ferla et al. 2002, Prinzing et al. 2003, Villaseñor et al. 2005, Posadas et al. 2006, Heino and Soininen 2007, Mandelik et al. 2007).

Looking for stable correlation structures independent of the adopted descriptors is a crucial step for any scientific field. Only robust structures obtained across different formalisation schemes can be considered consistent 'phase space' structures for the considered system (Hoover 1985, Broomhead and King 1986). Because the system studied here has a strong coupling between its elements (plant taxa), we can hypothesise that any sufficiently dense sampling of the floristic elements at different taxonomic levels (units, species, and genera) should keep track of the same underlying structure, as all levels are part of the same integrated system. In quantitative terms, this translates into looking for mutual correlations among the floristic distance matrices computed for Italian regions at different levels of taxonomic resolution. This invariant set of between regions floristic-based distances could then be considered an effective basis for Italy's phytogeographic description.

Thus, by using Italy and its native woody flora as a case study, we aimed to: 1) assess the basic invariance between different sampling strategies and identify the most explanatory variables; and 2) provide a phytogeographic regionalization for Italy, at a medium scale, on the basis of the identified explanatory variables, and detect correlations between the physical and biological factors shaping the identified phytogeographic structure. 


\section{Materials and methods}

Our study involved several steps concerning data sourcing and analysis (Fig. 1).

\subsection{Study area}

Italy is an interesting study case for the identification of biogeographic regions based on native woody flora due to its peculiar geographic location in the Mediterranean Sea, rich vascular flora, and highly diverse environments, including habitats (Viciani et al. 2014). Our previous study (Abbate et al. 2015) indicated that environmental heterogeneity of Italian regions rather than area is much more important for biodiversity, suggesting biodiversity is independent of the size of the analysed area.

Italy has an area of approximately $300,000 \mathrm{~km}^{2}$ comprising a continental northern sector, a peninsular centralsouthern sector, two large islands (Sardinia and Sicily), several archipelagos, and small islands. Its diverse physiography includes two major mountain ranges with different lithological features (the Alps, oriented East to West, and the Apennines, oriented Northwest to Southeast), volcanoes, and alluvial plains. A clear west to east gradient is evidenced from the Tyrrhenian to the Adriatic coast in terms of topography, lithology, soil type, and climate. Italy is classified into four climatic regions: Temperate, Mediterranean, and two transitional regions (Blasi \& Michetti 2007). The environmental heterogeneity of the Italian peninsula is due to its extension into the Mediterranean Sea, to the presence of latitudinal and longitudinal orographic systems, and to the influence of the large continental masses of Eurasia and Africa (Abbate et al. 2015). According to its latitudinal and elevation gradients, Italy should be almost completely covered by coniferous, hardwood, and sclerophyll forests, the exception being the alpine belt, psammophilous and halophilous coastal habitats, and lithosols (Blasi et al. 2010).

The biogeography of the Italian peninsula is the result of many palaeogeographic and palaeoclimatic events. During the Quaternary, several glacial cycles led to local extinction events, followed by rapid re-colonisation events. Italy played a key role in Europe's biogeography as, like other southern European peninsulas, it provided refuge for flora during the glacial periods (Svenning et al. 2008). The Mediterranean basin, a biodiversity hotspot of worldwide importance, is remarkable in terms of its plant species richness and endemism (Myers et al. 2000). 
Italy comprises 20 administrative regions, differentiated by their size, geography, geomorphology, bioclimate, and historical land use (Appendix), and mostly bordered by geographic discontinuities or catchment basins boundaries; hence, these regions represent an excellent natural grid to study Italy's biogeographic structure at a medium scale. Italian regions (and their acronyms) are: Abruzzo (ABR), Basilicata (BAS), Calabria (CAL), Campania (CAM), Emilia-Romagna (EMR), Friuli-Venezia Giulia (FVG), Lazio (LAZ), Liguria (LIG), Lombardia (LOM), Marche (MAR), Molise (MOL), Piemonte (PIE), Puglia (PUG), Sardegna (SAR), Sicilia (SIC), Toscana (TOS), Trentino-Alto Adige (TAA), Umbria (UMB), Valle d'Aosta (VDA), Veneto (VEN). Italy is an interesting study case for the identification of biogeographic regions based on native woody flora due to its peculiar geographic location in the Mediterranean Sea, rich vascular flora, and highly diverse environments, including habitats (Viciani et al. 2014). Our previous study (Abbate et al. 2015) indicated that environmental heterogeneity of Italian regions rather than area is much more important for biodiversity, suggesting biodiversity is independent of the size of the analysed area.

\subsection{Data sources}

The Italian native woody flora (trees, shrubs, lianas) dataset used here is the same as in Abbate et al. (2015). In particular, we superimposed the Raunkiaer system of life forms and subforms (Raunkiaer 1934, Pignatti 1982) over the species and subspecies (units) distribution data derived from Conti et al. (2005) and later updated by Conti et al. (2007) and VV. AA. (2005-2011), creating a woody plant data subset containing trees [Phanerophyte scapose (P scap) and Phanerophyte cespitose (P caesp)], shrubs [Nano-Phanerophyte (NP)], and lianas [Phanerophyte lianose (P lian)] presence/absence data within the 20 administrative regions. Unfortunately, a complete and updated floristic dataset for the entire country is only available at the regional scale (Abbate et al. 2015), reason why this scale was adopted.

Seven distance matrices were constructed based on the presence/absence dataset by applying the Hamming metric (Hamming 1950) to the following variables (vector spaces) within each of the 20 regions: a) whole woody flora-families $\left(\mathrm{WF}, \mathrm{n}^{\circ}\right.$ variables $\left.\left.=61\right), \mathrm{b}\right)$ genera $\left(\mathrm{WG}, \mathrm{n}^{\circ}\right.$ variables $\left.=133\right)$, c) phylogenetic relationships between genera $\left(\mathrm{PhG}, \mathrm{n}^{\circ}\right.$ variables $\left.=133\right)$, and $\left.\mathrm{d}\right)$ units (species and subspecies, $\mathrm{WU}, \mathrm{n}^{\circ}$ variables $=546)$; e) trees - families $\left(\mathrm{TF}, \mathrm{n}^{\circ}\right.$ variables $\left.\left.=19\right), \mathrm{f}\right)$ genera $\left(\mathrm{TG}, \mathrm{n}^{\circ}\right.$ variables $\left.=33\right)$, and $\left.\mathrm{g}\right)$ units $\left(\mathrm{TU}, \mathrm{n}^{\circ}\right.$ variables $\left.=124\right)$. As these were non-sparse matrices, using the Hamming metric provided an intuitive, 
unbiased, and suitable way to highlight the differences between the regions. Phylogenetic relationships between genera $(\mathrm{PhG})$ were obtained using the online software Phylomatic (http://phylodiversity.net/phylomatic/html/pm1.html), a tool allowing a rapid phylogenetic reconstruction among a set of taxa sparsely sampled from a large clade (Webb and Donoghue 2005), used in many studies (e.g. Ricotta et al. 2009). PhG assigned a weight to each region depending on the phylogenetic resemblance among the genera present within that region.

The seven data matrices (Mi), hereafter named 'sampling strategies', had the 20 Italian regions as statistical units (rows) and the floristic variables as columns. After applying Hamming's distance, each Mi provided an associated symmetric distance matrix (Di), having the 20 regions as rows and columns and their pairwise distances as the matrix elements. Our first step was to demonstrate the strong invariance among the pairwise distances obtained for the 20 regions (computed using the above data sets).

\subsection{Statistical methods}

\subsubsection{Correlation between distance spaces}

The amount of invariance between the seven sampling strategies (Mi) corresponds to the Pearson correlation coefficient between the distance matrices (Di) calculated for the sampling strategies. The components of these distance matrices are the $190(20 * 19 / 2)$ non-redundant distances between regions; the matrices can be comparated to each other after ordering them along consistent numbering. The higher the correlation coefficient, the greater the similarity between the corresponding distance matrices (Di) and the greater the coherence between the sampling strategies (Mi) (Randic et al. 1994).

In order to obtain an overview of the correlation between sampling strategies, using principal components analysis (PCA), we computed the eigenvectors of the distance between matrices and extracted the first principal component from the dataset. PCA results were further tested by the oblique principal components (OPC) method. OPC is a divisive variable clustering method in which variables are partitioned by maximising intra-cluster correlation; cluster formation ends when the correlation coefficient between clusters becomes significant, signalling that the clusters are not sufficiently differentiated (Giuliani et al. 2000). 


\subsubsection{Phytogeographic regionalization}

The distance matrix obtained for all woody genera (WG), which was the most representative of sampling strategies (see the results section), was then subjected to Kruskal-Wish multidimensional scaling (MDS) (Kruskal and Wish 1978). MDS projects statistical units (regions, in our case) as vector points in a twodimensional space, keeping the original pairwise distances between regions and allowing a topologically consistent representation of the floristic space. Correlations between the two spatial dimensions (mds1 and mds2), geographic coordinates (latitude, longitude), two new theoretical orographic indices (described below), and the amount of total plant diversity (also described below) were obtained using Pearson simple and partial correlation coefficients.

Latitude and longitude values of the barycentre of the different regions were correlated with mds 1 and mds2 to check for the relevance of geographic clines.

As shown in several studies (Currie and Paquin 1987, Field et al. 2007, Veetas and Ferrer-Castán 2008, Abbate et al. 2015), orographic variability scales with climatic variability. Therefore, in highly heterogeneous territories such as the Italian regions, where in a few kilometres sudden orographic and climatic changes occur, an index based on the amount of orographic and climatic internal variability across the three elevation belts would be more useful than average data. For this reason, two new theoretical 'orographic indices', named Distance from average ITAly (DITA) and Distance from SIMMetry (DSIMM), were used to track orographic variability. DITA and DSIMM values were based on the percentage cover of the three standard elevation belts (ISTAT 2011): plain (<300 m above sea level (a.s.1.)); hill (300-600 a.s.1.); and mountain ( $>600 \mathrm{~m}$ a.s.l.). The DITA value of each region was calculated as the Euclidean distance between the percentage cover of each of the three elevation belts and the average percentage cover of those belts in Italy ( $23 \%$ plains, $42 \%$ hills, $35 \%$ mountains). To evaluate DSIMM for each region, we calculated the Euclidean distance between the percentage cover of the three belts and the theoretically maximal symmetric configuration ( $33 \%$ plains, $33 \%$ hills, $33 \%$ mountains). DSIMM represents the distance from the maximal symmetry condition and hence from the highest Shannon entropy. On the sole basis of mathematical information theory (Shannon and Weaver 1949), maximal symmetry should correspond to the maximal biodiversity. On purely theoretical considerations, the maximal distance from maximal symmetry condition (high values of DSIMM) should correspond to a minimum in biodiversity, while low values of DSIMM 
should correspond to high biodiversity.

To analyse the amount of total plant diversity we used PC1BIODIV, a comprehensive index defined in Abbate et al. (2015). In detail, PC1BIODIV is the first component of a PCA (61\% of total variance) carried out using the Italian regions as the statistical objects, and the summary statistics for the total vascular flora and woody flora as variables (number of families, genera, species, units, and endemic units) (see Appendix for data). These PCA values were used as synthetic biodiversity scores within each region.

To delineate floristic homogeneous areas, the 20 regions were clustered in the two-dimensional space defined by the MDS axes using k-means clustering, the most popular non-hierarchical classification technique (Bishop 2005, Heikinheimo et al. 2007, Rueda et al. 2010). The results of the MDS scaling (region's scores on MDS axes) were submitted to k-means clustering instead of the original distance matrix because the MDS space almost completely (96\%) explained the original information regarding the distances between regions. In order to choose the optimal value of $\mathrm{k}$ (number of classes), we looked at the proportion of variance explained by the several partitions at increasing $\mathrm{k}$ values: a plateau in the curve relating the percentage of variance explained by the partition at increasing $\mathrm{k}$ marked the optimal structural classification of the data (Crescenzi and Giuliani 2001).

\section{Results}

\subsection{Correlation between distance spaces}

The seven different sampling strategies, based on different taxonomic levels, were strongly correlated with each other (Table 1). PhG was an exception as it presented moderate (but still highly significant) correlation coefficients with the other sampling choices. The high correlation $(r=0.939)$ between TU and WU was noteworthy.

Given the high correlation between variables, PCA's first component (PC1) explained $80 \%$ of the system's variability. The variables WG, TF, TU, and WU were the most related with PC1, while PC2 (explaining 10\% of total variance) accommodated the singular properties of PhG and WF (Table 2).

No statistically significant partition of the dataset was revealed by the OPC analysis applied to the seven distance matrices, with only one statistically significant class being found (data not shown).

Based on this important equivalence of the different sampling strategies, the subsequent analysis focused on 
WG, which was the variable most correlated with PC1 $(r=0.955)$ (Table 2), and thus, the most strongly associated with the underlying biogeographic structure.

\subsection{Phytogeographic regionalization}

The plot of the 20 regions in the space defined by mds 1 and mds2, resulting from the multidimensional scaling applied to WG's distance matrix, almost completely (96\%) explained the original information (Fig. 2a). The distribution of regions in the mds $1 / \mathrm{mds} 2$ two-dimensional space mirrored their relationships in terms of floristic patterns (Fig. 2a). The first axis (mds1) corresponds to the North-South gradient, as indicated by the high and significant correlation between mds1 and latitude $(r=0.932, p<0.0001)$ (Table 3, Fig. 2b); the second axis (mds2), although only moderately but significantly correlated with longitude $(\mathrm{r}=-0.624, p=$ 0.003) (Table 3), evidenced a less pronounced Eastern (Adriatic)-Western (Tyrrhenian) cline. The above hypothesis is supported by the correlation observed between latitude and longitude $(\mathrm{r}=-0.580, p=0.007)$ (Table 3) due to the North-West/South-East orientation of the Italian peninsula; the correlation between mds1 and longitude $(\mathrm{r}=-0.569, p=0.009)$ (Table 3$)$ is almost identical to that observed between latitude and longitude, suggesting mds1 can be interpreted as a pure North-South gradient.

A strong and significant correlation was found between DITA and DSIMM ( $\mathrm{r}=0.853, p<0.0001)$ (Table 3$)$, possibly due to the Italian territory presenting almost equal percentages coverage values of the three orographic belts.

The correlation between PC1BIODIV and DITA was significantly negative ( $\mathrm{r}=-0.645, p=0.002$, Fig. 3$)$; this correlation appears not to be influenced by the North-South gradient because it remained almost identical (Partial $\mathrm{r}=-0.612, p=0.007$ ) when the possible confounding effects of latitude and longitude were removed. The correlation between PC1BIODIV and DSIMM $(r=-0.549, p=0.012)$ was markedly lower than the one found between PC1BIODIV and DITA.

MDS (Fig. 2a) suggested a continuous cline instead of strongly individualised clusters. Notwithstanding that, we investigated the possibility of deriving a discrete partition of Italian regions into homogenous clusters on the $\mathrm{mds} 1 / \mathrm{mds} 2$-defined space. K-means clustering procedure suggested a six clusters solution of Italian regions $(\mathrm{k}=6 ; 90 \%$ of explained variance, Fig. 4). In addition to the dominant North-South axis, an Eastern 'Adriatic Apennine' (MAR, UMB, ABR, MOL, and BAS) and a Western 'Tyrrhenian Apennine' (LIG, TOS, 
LAZ, and CAM) sectors were identified. LOM, VEN, FVG, and EMR regions constituted the 'North-EasternPo valley' cluster, the 'purely Alpine' regions (VDA, PIE, and TAA) were identified as an independent group; PUG, CAL, and SIC clustered into a 'Southern' sector; SAR did not cluster with other regions because of its strong insularity.

Some relevant floristic patterns characterised the identified sectors. The highest value of woody genera richness was obtained in the 'Southern' sector (117 genera) followed by the 'Tyrrhenian Apennine' (116), 'Adriatic Apennine' (107), 'North-Eastern-Po valley' (93), and 'purely Alpine' (77) sectors; the minimum value was recorded in 'Sardinia' (30). Some genera were exclusive to a sector: Limonium was only found in 'Sardinia' and Fontanesia, Helianthemum, Ononis, Reaumuria, Retama, Rhumex, Rhus, Zelkova, and Ziziphus in the 'Southern' sector. On the other hand, many genera were present in all sectors except in 'Sardinia': Abies, Betula, Carpinus, Cercis, Cotoneaster, Cytisophyllum, Fagus, Humulus, Laburnum, Ligustrum, Loranthus, Staphylea, and Tilia.

\section{Discussion}

The present study identified Italy's phytogeographic structure at a medium scale, which was largely invariant across different sampling strategies. The strong correlation among the seven sampling strategies (data matrices) of the Italian native woody flora at the several taxonomic and life-form levels suggests the existence of a single hidden phase space defining the state of the system, which can be exhaustively described by a minimum number of variables (in this case woody taxa and, more specifically, woody genera distance). Once the invariance of Italy's phytogeographic characters was established, we were able to look for woody flora patterns and their correlation with orographic and geographic variables. As mentioned above, Italian regions have natural geographic borders (catchment basins, mountain ranges, sea) that allow considering them as proper divisions of the Italian territory. Our results confirm this hypothesis: the different sampling strategies of the regions gave a strongly invariant picture of Italy. Thus, the studied system is imposing its structure to the measurement paradigm, and not the other way around (Allen and Yen 2001), confirming the congruence of regional categorisation.

The level 'genera' (WG) appeared to be the explanatory variable that best described the woody flora pattern in the considered Italian regions, as it was the most correlated with the PCA's first component. Similar results 
for the level 'genera' have been found for phytogeographic regions in other continents (La Ferla et al. 2002) and for zoogeographic regions (Rueda et al. 2013), suggesting that knowledge at the species and subspecies level is often not indispensable for bioregionalization at a coarse scale. This result encourages the use of higher taxa for studying spatial patterns, especially in highly heterogeneous areas, diminishing the cost, time, and requirement for species experts in large-scale surveys; in fact, many authors have already remarked the reduction in costs when applying coarse taxonomic resolution for conservation purposes (Heino and Soininen 2007, Mandelik et al. 2007).

The use of the dataset 'native woody flora' as a shortcut to study biodiversity was also validated by the high correlation between TU and WU (Table 1) in terms of qualitative data; this result is consistent with previous findings regarding the correlation between the amount of woody units (trees in particular) and total vascular plant richness (Abbate et al. 2015).

The phylogenetic genera diversity $(\mathrm{PhG})$ resulted to be less informative than family, genera, and units diversity for explaining Italy's current floristic patterns, suggesting complex palaeogeographic, palaeoclimatic, and human-related events have shaped the phylogeny of Italian flora (Blasi et al. 2007, Magri 2008, Svenning et al. 2008). Notwithstanding, the moderate correlation values found between PhG and other sampling strategies might be used to perform a coarse assessment of the effects of history on current woody flora distribution.

A latitudinal cline in the woody genera distribution along the Italian regions was evidenced by the regional distribution along the first ordination axis (mds1), that followed a North-South gradient, and by the strong correlation found between mds1 and latitude. A longitudinal cline was also present, as indicated by the significant correlation found between mds2 and longitude, distinguishing the bioclimatic conditions existing between the western-Tyrrhenian and eastern-Adriatic sides of peninsular Italy. All these features are congruent with the bioclimatic framework and vegetation series described by Blasi and Michetti (2007) and by Blasi (2010).

The positively biased Italian orography towards hills ( $42 \%$ of hills instead of $33 \%$ ) has led to a higher biodiversity than that expected under a theoretical maximum symmetry of altimetric belt. In fact, DITA was more correlated than DSIMM to PC1BIODIV. This was an indirect proof of hill's higher relevance for defining biodiversity, expressed by vascular species richness, compared to plains and mountains. A very high 
vegetation diversity in Italian hills was also observed by Blasi (2010). Thus, the index DITA might be useful tool to analyse floristic orographic patterns. Altitude range, being a surrogate for climatic heterogeneity, has an important and direct effect on flora distribution, regardless of the geographic clines, as already demonstrated in other studies (Moreno Saiz and Lobo 2008, Veetas and Ferrer-Castán 2008, Grau et al. 2012). For the Balkans, tree species richness was shown to be strongly correlated with the elevation range and weakly correlated with rainfall (Field et al. 2007). Recent landscape classification of the Czech Republic, based on the distribution of the natural habitats, showed that cluster separation is primarily determined by altitude and related climatic factors (Divíšek et al. 2014). At the European scale, animals distribution has also been correlated with topography and climate (Heikinheimo et al. 2007, Rueda et al. 2010).

The woody genera distribution along geographic clines, where neighbouring regions showed no noticeable discontinuity between them, explained why Italian regions were not very strongly separated. However, the Kmeans clustering showed a separation between northern (alpine) and peninsular Italy. The separation between alpine and peninsular regions was also highlighted in the clusters obtained by Rueda et al. (2010) based on tree flora, by Bohn and Neuhäusl (2000/2003) based in forest types, by Pignatti (2011) and by Peruzzi et al. (2014) considering the entire Italian endemic flora (1371 species and subspecies). The regionalization based on woody genera presented in the present study was also able to distinguish the 'purely Alpine' from the 'Alpine-Po Valley' sector and to identify Sardinia's strong insularity. The lack of a clear discontinuity between clusters can be specifically linked to the 13 genera shared by all groups except 'Sardinia'. A geographic cline was also evidenced by woody genera richness as the number of genera strongly increased from the 'purely Alpine' to the 'Southern' sector. The peculiarity of the 'Southern' sector was also marked by the presence of nine exclusive genera, most including endemic or Mediterranean species. The strong insularity of 'Sardinia' was more related to the lack of many genera than to the presence of exclusive ones.

Despite the adopted scale, our regionalization is consistent with that stated by other authors (Giacomini 1958, Takhtajan 1986, Rivas-Martinez 2004). In particular, Giacomini (1958) recognised two provinces within the central European region (with five districts), called 'Alpine Province' and 'Apennine Province', and two provinces within the Mediterranean Region (with five districts) called 'Adriatic Province' and 'LigurianTyrrhenian Province'. Takhtajan's (1986) later map largely overlapped with Giacomini provinces. The regionalization proposed by Rivas-Martinez (2004) only diverges from this model regarding the extent of the 
Apennine province in order to comprise the Southern Apennines in the Apennine sector. Giacomini (1958), Rivas-Martinez (2004), Arrigoni (2006) and, more recently, Peruzzi et al. (2014), have all pointed out the peculiar phytogeographic structure of Sardinia, characterised by a rich Sardinian and Sardinian-Corsican endemic taxa, classifying it as an autonomous sector. In conclusion, the phytogeographic structure of Italy can be detected using only a few variables, like woody genera (133 variables), as indicated by the overlapping of our results with these ones. Our regionalization adds useful insight into the country's biogeographic patterns to the previous biogeographic classifications.

Unfortunately, the available database for the administrative regions used here did not allow further distinguishing of sub-sectors, as far as peninsular Italy is concerned, within the Mediterranean and temperate Italian regions. For areas with high environmental heterogeneity such as Italy, the next step will be to test our method at a finer scale and to identify phytogeographic sub-sectors, surely more appropriate for conservation purposes. As recently pointed out by some authors, regionalization 'holds continuing relevance to pure and applied biogeography' (Ladle and Whittaker 2011, Whittaker et al. 2013).

\section{Acknowledgements}

We thank the two anonymous reviewers and the journal editors for their interesting and valuable comments on the manuscript. 


\section{References}

Abbate, G., Bonacquisti, S., Burrascano, S., Giovi, E., Giuliani, A., Pretto, F. and Scassellati, E. 2015. Woody flora as a predictor of vascular plant richness: an insight in Italy. Plant Biosyst. 149(3): 565-573. doi: $10.1080 / 11263504.2013 .870251$.

Allen, M.J. and Yen W.M. 2001. Introduction to Measurement Theory, Waveland Press, Inc. Long Growe, Illinois.

Arrigoni, P.V. 2006. Flora dell'isola di Sardegna, Carlo Delfino, Sassari.

Bishop, C.M. 2005. Neural networks for pattern recognition, Oxford University Press, New York.

Blasi, C. (Editor). 2010. La vegetazione d'Italia, Palombi \& Partner S.r.l., Roma, 538 pp.

Blasi, C., Capotorti, G., Copiz, R., Guida, D., Mollo, B., Smiraglia, D. and Zavattero, L. 2014. Classification and mapping of the ecoregions of Italy. Plant Biosyst. 148(6): 1255-1345. doi:

$10.1080 / 11263504.2014 .985756$.

Blasi, C., Filibeck, G. and Vigna Taglianti, A. 2007. Biodiversity and biogeography. In Biodiversity in Italy. Contribution to the National Biodiversity Strategy. Edited by C. Blasi, L. Boitani, S. La Posta, F. Manes, M. Marchetti. Palombi Editori, Roma. pp. 40-56. Available from: http://www.minambiente.it/sites/default/files/archivio/biblioteca/protezione_natura/dpn_biodiversita_italia_en g.zip (accessed: 30 September 2015).

Blasi, C. and Michetti, L. 2007. Biodiversity and climate. In Biodiversity in Italy. Contribution to the National Biodiversity Strategy. Edited by C. Blasi, L. Boitani, S. La Posta, F. Manes, M. Marchetti. Palombi Editori, Roma. pp. 57-66. Available from:

http://www.minambiente.it/sites/default/files/archivio/biblioteca/protezione_natura/dpn_biodiversita_italia_en g.zip (accessed: 30 September 2015).

Bohn, U. and Neuhäusl, R. 2000/2003. Karte der natürlichen Vegetation Europas. (Map of the Natural Vegetation of Europe). Landwirtschaftsverlag, Münster. Maßstab/Scale 1: 250000.

Broomhead, D.S. and King, G.P. 1986. Extracting qualitative dynamics from experimental data. Phys. D 20: 217-236.

de Candolle, A. 1855. Geographie botanique raisonée. Librairie de Victor Masson, Paris. 
Conti, F., Abbate, G., Alessandrini, A. and Blasi, C. (Editors). 2005. An annotated checklist of the Italian vascular flora. Palombi Editori, Roma.

Conti F., Alessandrini A., Bacchetta G., Banfi E., Barberis G., Bartolucci F., Bernardo L., Bonacquisti S., Bouvet D., Bovio M., Brusa G., Del Guacchio E., Foggi B., Frattini S., Galasso G., Gallo L., Gangale C., Gottschlich G., Grünanger P., Gubellini L., Iiriti G., Lucarini D., Marchetti D., Moraldo B., Peruzzi L., Poldini L., Prosser F., Raffaelli M., Santangelo A., Scassellati E., Scortegagna S., Selvi F., Soldano A., Tinti D., Ubaldi D., Uzunov D. and Vidali M. 2007. Integrazioni alla Checklist della flora vascolare italiana. Natura Vicentina 10 (2006): 5-74.

Crescenzi, M. and Giuliani, A. 2001. The main biological determinants of tumor line taxonomy elucidated by a principal component analysis of microarray data. FEBS Letters 507: 114-118.

Currie, D.J. and Paquin, V. 1987. Large-scale biogeographical patterns of species richness of trees. Nature 24: 326-327. doi:10.1038/329326a0.

European Environment Agency 2012. Biogeographic regions in Europe [online]. Available from http://www.eea.europa.eu/data-and-maps/figures/biogeographical-regions-in-europe-1 [accessed 1 October 2015].

Divíšek, J., Chytrý, M., Grulich, V. and Poláková, L. 2014. Landscape classification of the Czech Republic based on the distribution of natural habitats. Preslia 86: 209-231.

Field, R., O’Brien, E.M. and Lavers, C.P. 2007. Global models for predicting woody plant richness from climate: reply. Ecology 88(1): 259-272. doi.org/10.1890/04-1910.

Giacomini, V. 1958. Conosci l'Italia: La Flora. TCI, Milano.

Giuliani, A., Benigni, R., Sirabella, P., Zbilut, J.P. and Colosimo, A. 2000. Nonlinear Methods in the analysis of protein sequences. A case study on rubredoxins. Biophys. J. 78(1): 136-148. doi:10.1016/S0006$3495(00) 76580-5$.

ISTAT 2011. Annuario Statistico Italiano. Roma. p. 19.

Grau, O., Ninot. J.M., Ferré, A., Font, X. and Grytnes J. 2012. Altitudinal species richness patterns of vascular plants in the South-Eastern Pyrenees and nearby mountains of Catalonia. Plant Ecol. Divers. 5(1): 1-12. doi: 10.1080/17550874.2012.666027.

Hamming, R. W. 1950. Error detecting and error correcting codes. Bell Syst. Tech. J. 29(2): 147-160. 
doi:10.1002/j.1538-7305.1950.tb00463.x

Heikinheimo, H., Fortelius, M., Eronen, J. and Mannila, H. 2007. Biogeography of European land mammals shows environmentally distinct and spatial coherent clusters. J. Biogeogr. 34(6): 1053-1064.

doi: 10.1111/j.1365-2699.2006.01664.x.

Heino, J. and Soininen, J. 2007. Are higher taxa adequate surrogates for species-level assemblage patterns and species richness in stream organims? Biol. Conserv. 137(1) 78-89. doi:10.1016/j.biocon.2007.01.017.

Hoover, W.G. 1985. Canonical dynamics: Equilibrium phase-space distributions. Phys. Rev. A 31(3): 16951697.

Kreft, H. and Jetz, W. 2010. A framework for delineating biogeographical regions based on species distributions. J. Biogeogr. 37(11): 2029-2053. doi: 10.1111/j.1365-2699.2010.02375.x.

Kruskal, J.B. and Wish, M. 1978. Multidimensional Scaling. Sage Publications, Newbury Park, CA. von Humboldt, A. 1806. Essai sur la geographie des plantes; accompagné d'un tableau physique des région équinoxales, accompagné d'un tableau physique des région équinoctiales. Schoel \& Co., Paris.

Ladle, R.J. and Whittaker, R.J. (Editors). 2011. Conservation Biogeography. Wiley-Blackwell, Oxford. La Ferla, B., Taplin, J., Ockwell, D. and Lovett, J.C. 2002. Continental scale patterns of biodiversity: can higher taxa accurately predict African plant distributions? Bot. J. Linn. Soc. 138: 225-235.

Magri, D. 2008. Pattern of post-glacial spread and the extent of glacial refugia of European beech (Fagus sylvatica). J. Biogeogr. 35(3): 450-463. doi: 10.1111/j.1365-2699.2007.01803.x.

Mandelik, Y., Dayan, T., Chikatunov, V. and Kravchenko, V. 2007. Reliability of a higher-taxon approach to richness, rariry and composition assessments at the local scale. Conserv. Biol. 21(6): 1506-1515. doi: 10.1111/j.1523-1739.2007.00823.x.

Moreno Saiz, J.C. and Lobo, J.M. 2007. Iberian-Balearic fern regions and their explanatory variables. Plant Ecol. 198: 149-167. doi: 10.1007/s11258-007-9392-8.

Myers, N., Mittermeier, R.A., Mittermeier, C.G., da Fonseca, G.A.B. and Kent, J. 2000. Biodiversity hotspots for conservation priorities. Nature 403: 853-858. doi: 10.1038/35002501.

Olson, D.M., Dinerstein, E., Wikramanayake, E.D., Burgess, N.D., Powell, G.V.N., Underwood, E.C., D’Amico, J.A., Strand, H.E., Morrison, J.C., Loucks, C.J., Allnutt, T.F., Lamoreux, J.F., Ricketts, T.H., Itoua, 
I., Wettengel, W.W., Kura, Y., Hedao, P. and Kassem, K. 2001. Terrestrial ecoregions of the world: a new map of life on Earth. BioScience 51(11): 933-938.

Peruzzi, L., Conti, F. and Bartolucci, F. 2014. An inventory of vascular plants endemic to Italy. Phytotaxa 168(1): 1-75. doi: http://dx.doi.org/10.11646/phytotaxa.168.1.1.

Pignatti G. 2011. La vegetazione forestale di fronte ad alcuni scenari di cambiamento climatico in Italia.

Forest@ 8: 1-12. doi: 10.3832/efor0650-008.

Pignatti, S. 1982. Flora d'Italia. Edagricole, Bologna.

Posadas, P., Crisci, J.V. and Katinas, L. 2006. Historical biogeography: a review of its basic concepts and critical issues. J. Arid Environ. 66: 389-403. doi:10.1016/j.jaridenv.2006.01.004.

Prinzing, A., Klotz, S., Stadler, J. and Brandl, R. 2003. Woody plants in Kenya: expanding the higher-taxon approach. Biol. Conserv. 110(3): 307-314. doi:10.1016/S0006-3207(02)00242-2.

Randic, M., Kleiner, A.F. and De Alba, L.M. 1994. Distance/distance matrixes. J. Chem. Inf. Comp. Sci. 34: 277-286.

Raunkiaer, C. 1934. The life forms of plants and statistical plant geography. Oxford University Press, Oxford. Ricotta, C., La Sorte, F.A., Pysek, P., Rapson, G.L., Celesti-Grapow, L. and Thompson, K. 2009.

Phyloecology of urban alien floras. J. Ecol. 97(6): 1243-1251. doi: 10.1111/j.1365-2745.2009.01548.x.

Rivas-Martinez, S., Penas, A. and Diaz, T.E. 2004. Biogeographic maps of Europe. Cartographic Service, University of León, Spain. Available from: http://www.globalbioclimatics.org/form/bg med.htm (accessed: 30 September 2015).

Rueda, M., Rodríguez, M.Á. and Hawkins, B.A. 2010. Towards a biogeographic regionalization of the European biota. J. Biogeogr. 37(11): 2067-2076. doi: 10.1111/j.1365-2699.2010.02388.x.

Rueda, M., Rodríguez, M.Á. and Hawkins, B.A. 2013. Identifying global zoogeographical regions: lessons from Wallace. J. Biogeogr. 40(12): 2215-2225. doi: 10.1111/jbi.12214.

Shannon, C.E. and Weaver, W. 1949. The mathematical theory of Communication. University of Illinois Press, Urbana.

Svenning, J.C., Normand, S. and Kageyama, M. 2008. Glacial refugia of temperate trees in Europe: insights from species distribution modelling. J. Ecol. 96(6): 1117-1127. doi: 10.1111/j.1365-2745.2008.01422.x. 
Takhtajan, A.L. 1986. Floristic regions of the world. University of California Press, Berkley-Los AngelesLondon.

Veetas, O.R. and Ferrer-Castán, D. 2008. Patterns of woody plant species richness in the Iberian Peninsula: environmental range and spatial scale. J. Biogeogr. 35(10): 1863-1878. doi: 10.1111/j.13652699.2008.01931.x.

Viciani, D., Lastrucci, L. and Dell'Olmo, L. 2014. Natura 2000 habitats in Tuscany (central Italy): synthesis of main conservation features based on a comprehensive database. Biodivers. Conserv. 23: 1551-1576. doi: 10.1007/s10531-014-0686-6.

Villaseñor, J.L., Ibarr-Manríquez, G., Meave, J.A. and Ortíz, E. 2005. Higher taxa as surrogates of plant biodiversity in a megadiverse country. Conserv. Biol. 19(1): 232-238. doi: 10.1111/j.1523-1739.2005.00264.x VV.AA. 2005-2011. Notulae alla checklist della flora vascolare italiana. Inform. Bot. Ital. 37-43.

Wallace, A.R. 1876. The geographical distribution of animals. Harper, New York.

Webb, C.O. and Donoghue, M.J. 2005. Phylomatic: tree assembly for applied phylogenetics. Mol. Ecol. Notes 5: 181-183. doi: 10.1111/j.1471-8286.2004.00829.x Whittaker, R.J., Riddle, B.R., Hawkins, B.A. and Ladle, R.J. 2013. The geographical distribution of life and the problem of regionalization: 100 years after Alfred Russell Wallace. J. Biogeogr. 40(12): 2209-2214. doi: $10.1111 /$ jbi.12235. 


\section{Table 1}

\begin{tabular}{|l|l|l|l|l|l|l|l|}
\hline & WF & WG & PhG & WU & TF & TG & TU \\
\hline WF & 1.000 & & & & & & \\
\hline WG & $\mathbf{0 . 8 2 2}$ & 1.000 & & & & & \\
\hline PhG & 0.789 & 0.734 & 1.000 & & & & \\
\hline WU & 0.663 & $\mathbf{0 . 9 1 0}$ & 0.576 & 1.000 & & & \\
\hline TF & 0.666 & $\mathbf{0 . 8 5 0}$ & 0.612 & $\mathbf{0 . 9 3 9}$ & 1.000 & & \\
\hline TG & 0.620 & 0.792 & 0.565 & 0.764 & $\mathbf{0 . 8 0 1}$ & 1.000 & \\
\hline TU & 0.666 & $\mathbf{0 . 8 5 0}$ & 0.612 & $\mathbf{0 . 9 3 9}$ & $\mathbf{1 . 0 0 0}$ & $\mathbf{0 . 8 0 1}$ & 1.000 \\
\hline
\end{tabular}

Pearson's correlation coefficients between the distances relative to the different sampling strategies (acronyms are defined in the text, bold face values correspond to the most mutually consistent sampling pairs). 
Table 2

\begin{tabular}{|l|l|l|}
\hline & PC1 & PC2 \\
\hline WF & $\mathbf{0 . 8 2 9}$ & $\mathbf{0 . 4 5 1}$ \\
\hline WG & $\mathbf{0 . 9 5 5}$ & 0.064 \\
\hline PhG & 0.770 & $\mathbf{0 . 5 5 2}$ \\
\hline WU & $\mathbf{0 . 9 3 5}$ & -0.247 \\
\hline TF & $\mathbf{0 . 9 4 6}$ & -0.254 \\
\hline TG & $\mathbf{0 . 8 5 7}$ & -0.173 \\
\hline TU & $\mathbf{0 . 9 4 6}$ & -0.254 \\
\hline
\end{tabular}

PCA components loading matrix (bold face values highlight the most relevant variables for interpreting the corresponding component). 
Table 3

\begin{tabular}{|l|l|l|l|l|l|l|}
\hline & mds1 & mds2 & latitude & longitude & DITA & DSIMM \\
\hline mds1 & 1.000 & & & & & \\
\hline mds2 & 0.000 & 1.000 & & & & \\
\hline latitude & $\mathbf{0 . 9 3 2}$ & 0.181 & 1.000 & & & \\
\hline longitude & $\mathbf{- 0 . 5 6 9}$ & $\mathbf{- 0 . 6 2 4}$ & $\mathbf{- 0 . 5 8 0}$ & 1.000 & & \\
\hline DITA & $\mathbf{0 . 5 4 8}$ & -0.126 & $\mathbf{0 . 5 4 1}$ & -0.335 & 1.000 & \\
\hline DSIMM & 0.256 & -0.067 & 0.208 & -0.182 & $\mathbf{0 . 8 5 3}$ & 1.000 \\
\hline
\end{tabular}

Pearson's correlation coefficients between MDS axes (computed using WU), geographic, and orographic variables (acronyms are defined in the text). 


\section{Figure captions}

Fig. 1. Conceptual diagram of the steps involved in data sourcing and statistical analyses (numbers correspond to the subparagraphs).

Fig. 2. a) Multidimensional scaling based on the distance matrix of the woody genera (WG), mirroring the spatial relationships between the Italian regions (acronyms are defined in the text).

b) Multidimensional scaling showing the correlation between latitude and mds1 for the Italian regions.

Fig. 3. Correlation between DITA and PC1BIODIV (acronyms are defined in the text).

Fig. 4 Clustering of Italian regions into six groups (90\% of explained variance) (acronyms are defined in the text; administrative boundaries data from http://www.istat.it/it/archivio/124086). 


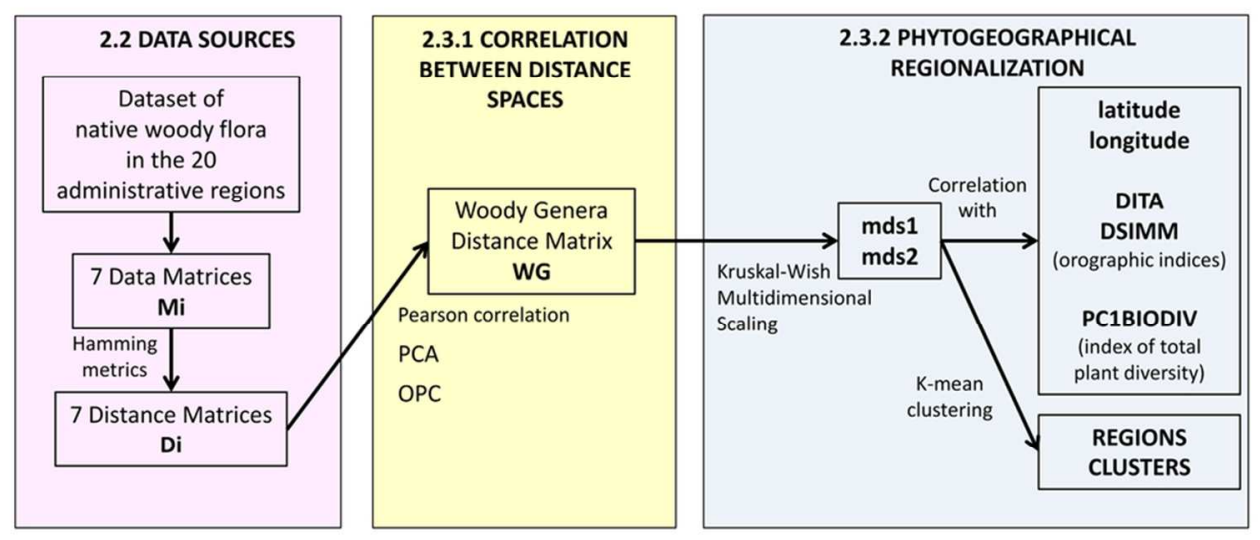

Fig. 1. Conceptual diagram of the steps involved in data sourcing and statistical analyses (numbers correspond to the subparagraphs).

$86 \times 40 \mathrm{~mm}(300 \times 300$ DPI $)$ 

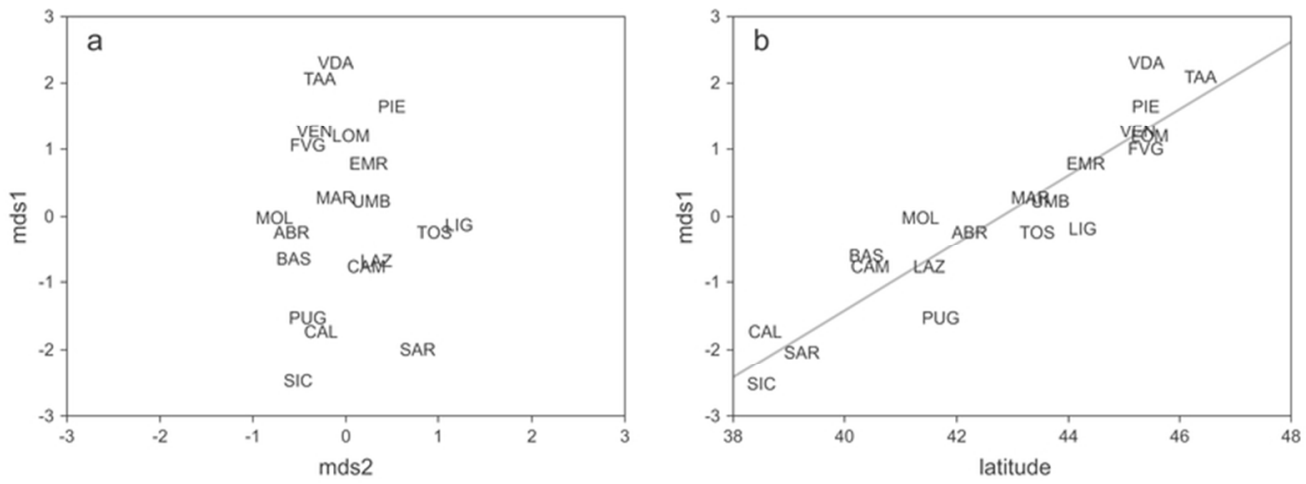

Fig. 2. a) Multidimensional scaling based on the distance matrix of the woody genera (WG), mirroring the spatial relationships between the Italian regions (acronyms are defined in the text). $67 \times 24 \mathrm{~mm}(300 \times 300$ DPI) 


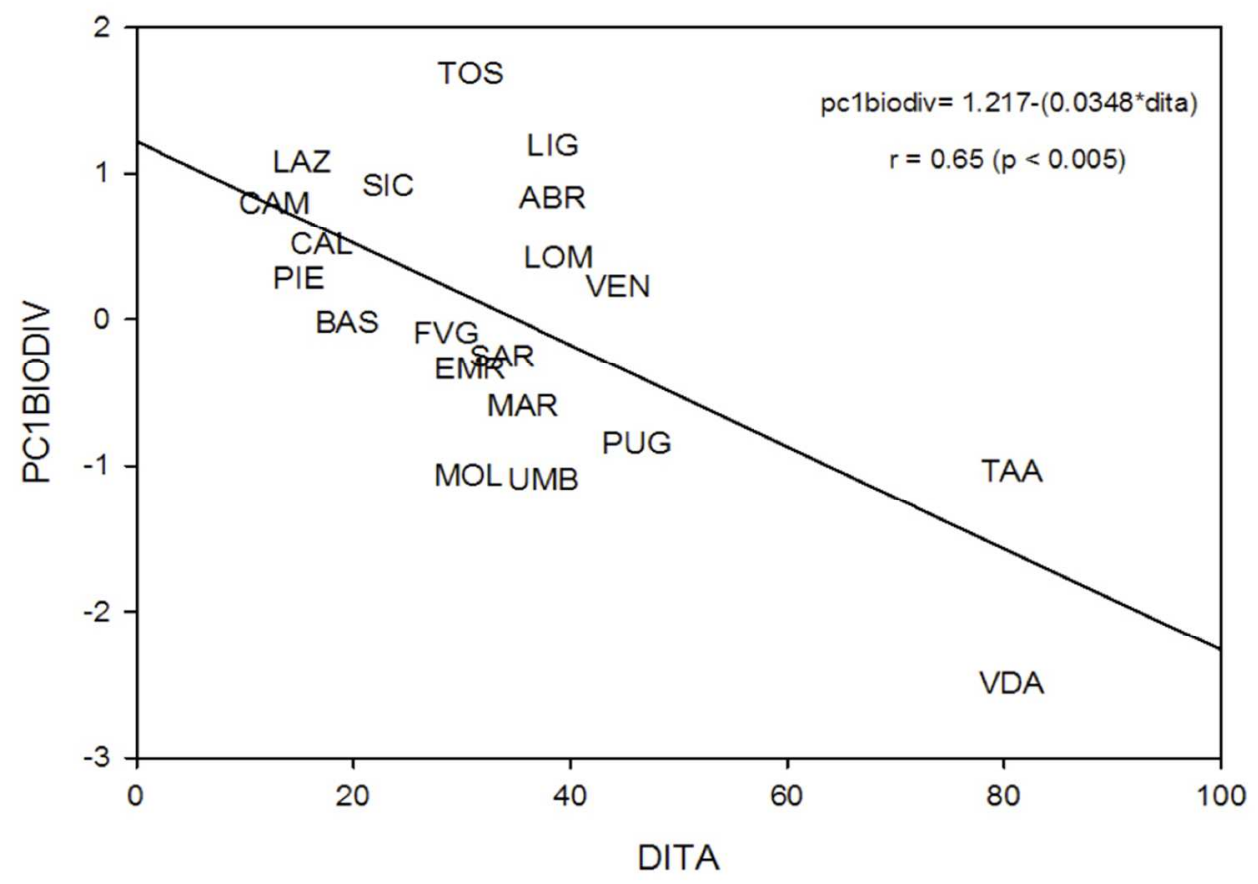

Fig. 3. Correlation between DITA and PC1BIODIV (acronyms are defined in the text). $86 \times 62 \mathrm{~mm}(300 \times 300 \mathrm{DPI})$ 

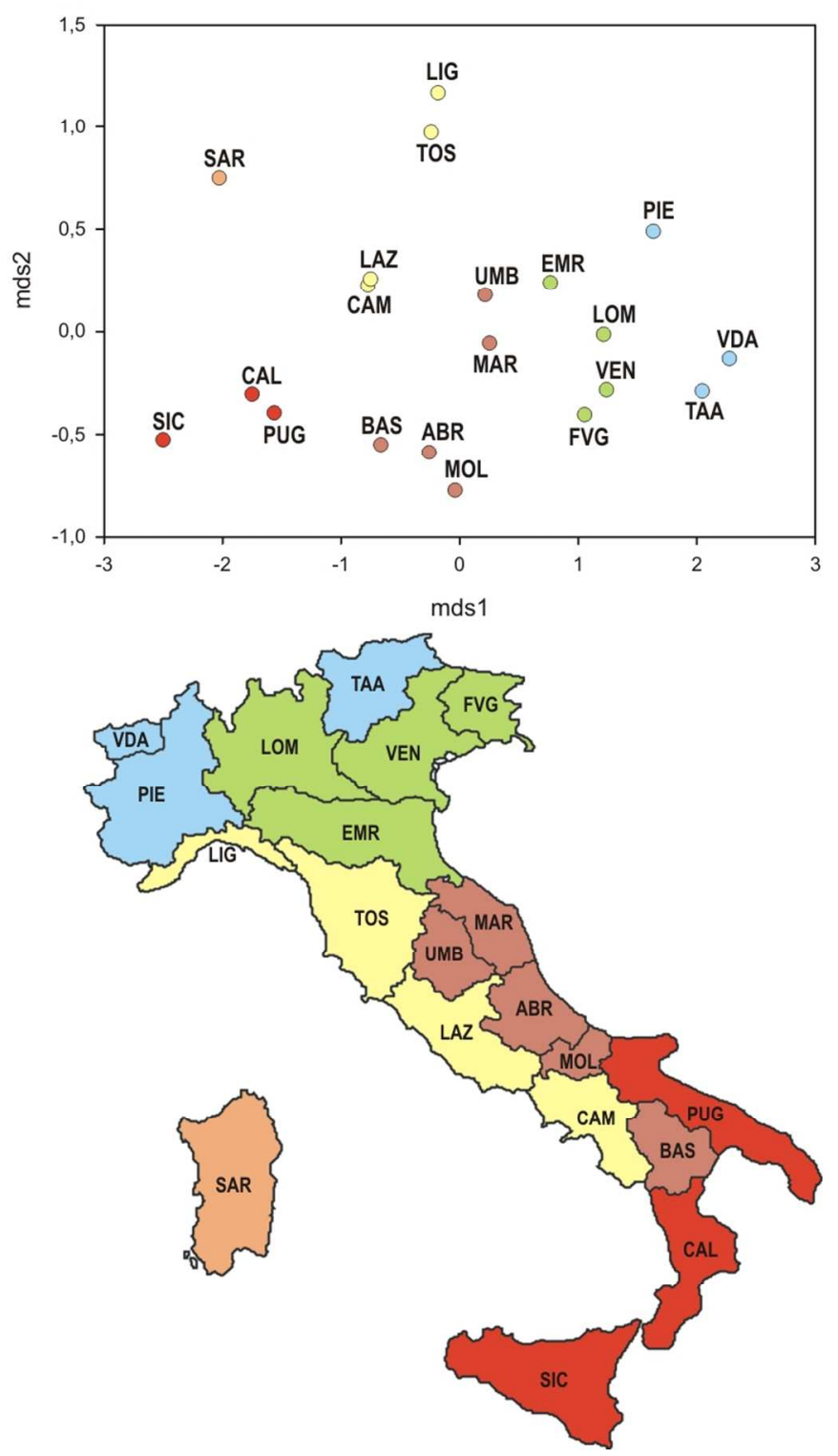

Fig. 4 Clustering of Italian regions into six groups (90\% of explained variance) (acronyms are defined in the text; administrative boundaries data from http://www.istat.it/it/archivio/124086) $76 \times 122 \mathrm{~mm}(300 \times 300$ DPI $)$ 


\begin{tabular}{|c|c|c|c|c|c|c|c|c|c|c|}
\hline Regions & mds1 & mds2 & pc1 biodiv & Latitude & Longitude & $\%$ plain & $\%$ hill & $\%$ mountain & DITA & DSIMM \\
\hline ABR & -0.252669 & -0.581692 & 0.829184606 & 42.21 & 13.23 & 0 & 35 & 65 & 38.44477 & 46.01087 \\
\hline BAS & -0.663934 & -0.550174 & -0.024608092 & 40.38 & 15.48 & 8 & 45 & 47 & 19.44222 & 31.06445 \\
\hline CAL & -1.746481 & -0.302223 & 0.504465449 & 38.53 & 16.35 & 9 & 49 & 42 & 17.14643 & 30.21589 \\
\hline CAM & -0.773732 & 0.229409 & 0.790512257 & 40.5 & 14.15 & 14 & 51 & 35 & 12.72792 & 26.24881 \\
\hline EMR & 0.773436 & 0.24251 & -0.342858387 & 44.3 & 10.57 & 48 & 27 & 25 & 30.82207 & 18.02776 \\
\hline FVG & 1.064885 & -0.402908 & -0.104934188 & 45.38 & 13.48 & 38 & 19 & 43 & 28.6007 & 17.91647 \\
\hline LAZ & -0.7537 & 0.25883 & 1.073370632 & 41.53 & 12.28 & 20 & 54 & 26 & 15.29706 & 25.671 \\
\hline LIG & -0.180797 & 1.170906 & 1.184333789 & 44.27 & 8.44 & 0 & 35 & 65 & 38.44477 & 46.01087 \\
\hline LOM & 1.21403 & -0.014087 & 0.410657799 & 45.35 & 9.55 & 47 & 12 & 41 & 38.88444 & 26.4764 \\
\hline MAR & 0.249397 & -0.050518 & -0.599871276 & 43.37 & 13.31 & 0 & 69 & 31 & 35.69314 & 48.8774 \\
\hline $\mathrm{MOL}$ & -0.03449 & -0.775422 & -1.065380843 & 41.34 & 14.4 & 0 & 45 & 55 & 30.62679 & 41.4367 \\
\hline PIE & 1.639946 & 0.489341 & 0.274402029 & 45.4 & 7.42 & 27 & 30 & 43 & 14.96663 & 12.04159 \\
\hline PUG & -1.559545 & -0.393827 & -0.85093174 & 41.7 & 16.52 & 54 & 45 & 1 & 46.10857 & 40.11234 \\
\hline SAR & -2.024864 & 0.743611 & -0.264404297 & 39.13 & 9.7 & 18 & 68 & 14 & 33.79349 & 42.55585 \\
\hline SIC & -2.502532 & -0.524329 & 0.916533192 & 37.33 & 14.08 & 14 & 61 & 25 & 23.28089 & 34.77068 \\
\hline TAA & 2.051646 & -0.289776 & -1.039457583 & 46.4 & 11.7 & 0 & 0 & 100 & 80.73413 & 81.6517 \\
\hline TOS & -0.239639 & 0.974575 & 1.67966186 & 43.46 & 11.15 & 8 & 67 & 25 & 30.82207 & 42.95346 \\
\hline UMB & 0.2156 & 0.18628 & -1.095793898 & 43.63 & 12.23 & 0 & 71 & 29 & 37.49667 & 50.48762 \\
\hline VDA & 2.279975 & -0.131344 & -2.495054314 & 45.4 & 7.2 & 0 & 0 & 100 & 80.73413 & 81.6517 \\
\hline VEN & 1.243467 & -0.27916 & 0.220173004 & 45.26 & 12.19 & 57 & 14 & 29 & 44.45222 & 30.8707 \\
\hline
\end{tabular}

Appendix A Table 1. Dataset used in this study (acronyms are defined in the text).

Legend

Regions: the 20 administrative regions of Italy

mds1: distance information in first resulting spatial dimensions of Multidimensional Scaling

mds2: distance information in second resulting spatial dimensions of Multidimensional Scaling

pc1 biodiv: synthetic biodiversity score computed by means of PCA

Latitude (midpoint): latitude midpoint of the 20 administrative regions of Italy

Longitude (midpoint): longitude midpoint of the 20 administrative regions of Italy

$\%$ plain, $\%$ hill and $\%$ mountain: percentage cover of the 3 standard elevation belts

DITA, DSIMM: orographic indexes (see text) 